\title{
The length of the second fundamental form, a tangency principle and applications
}

\author{
FRANCISCO X. FONTENELE ${ }^{1}$ and SÉRgIO L. SILVA ${ }^{2}$ \\ ${ }^{1}$ Universidade Federal Fluminense, Instituto de Matemática, Departamento de Geometria \\ 24020-140 Niterói, RJ, Brasil \\ ${ }^{2}$ Universidade Estadual do Rio de Janeiro-UERJ, Departamento de Estruturas Matemáticas-IME \\ 20550-013 RJ, Brasil
}

Manuscript received on September 5, 2003; accepted for publication on November 21, 2003; presented by MANFREDO DO CARMO

\begin{abstract}
In this paper we prove a tangency principle (see Fontenele and Silva 2001) related with the length of the second fundamental form, for hypersurfaces of an arbitrary ambient space. As geometric applications, we make radius estimates of the balls that lie in some component of the complementary of a complete hypersurface into Euclidean space, generalizing and improving analogous radius estimates for embedded compact hypersurfaces obtained by Blaschke, Koutroufiotis and the authors. The basic tool established here is that some operator is elliptic at points where the second fundamental form is positive definite.
\end{abstract}

Key words: hypersurfaces, tangency principle, second fundamental form, balls, radius estimates.

\section{INTRODUCTION}

In (Fontenele and Silva 2001), the same authors proved that if an $n$-dimensional embedded compact hypersurface $M^{n}$ into $(n+1)$-dimensional Euclidean space satisfies $\left|H_{k}\right| \geq \frac{1}{\lambda^{k}}$, for some $k$-mean curvature $H_{k}, 1 \leq k \leq n$, and some positive constant $\lambda$, then the greatest ball that fits inside $M^{n}$ has radius less than $\lambda$ unless $M^{n}$ is a sphere of radius $\lambda$, generalizing results of (Blaschke 1956) and (Koutroufiotis 1973) for surfaces. Our basic tool for the proof of the above result was a tangency principle stated in (Fontenele and Silva 2001) as Theorem 1.1. This tangency principle turned out to be very useful to obtain other geometric applications. In this work we obtain a tangency principle (see Fontenele and Silva 2001) related with the length of the second fundamental form and improve

The first author dedicates this work to his parents José (in memoriam) and Herondina

Correspondence to: Francisco X. Fontenele

E-mail: fontenele@mat.uff.br 
(see Remark 3.1) the radius estimate for the greatest ball that fits inside a suitable component of the complementary of an $n$-dimensional complete hypersurface into $(n+1)$-dimensional Euclidean space. In order to state our results we need the following.

As in (Fontenele and Silva 2001), given a hypersurface $M^{n}$ of a complete Riemannian manifold $N^{n+1}$ with metric $\langle$,$\rangle and exponential mapping exp: T N \rightarrow N$, we parametrize a neighborhood of $M^{n}$ containing $p$ and contained in a normal ball of $N^{n+1}$ putting

$$
\varphi(x)=\exp _{p}\left(x+\mu(x) \eta_{o}\right)
$$

where $x$ varies in a neighborhood $W$ of zero in $T_{p} M$ (the tangent space to $M^{n}$ at $p$ ), $\eta_{o}$ is a fixed unitary vector normal to $M^{n}$ at $p$ and $\mu$ is an unique real function defined in $W$ with $\mu(0)=0$. Let $\eta: W \rightarrow T_{\varphi(W)}^{\perp} M$ be a local orientation of $M^{n}$ with $\eta(0)=\eta_{o}$. Denote by $A_{\eta(x)}$ the second fundamental form of $M^{n}$ in the direction $\eta(x)$ and by $\sigma$ the vector valued second fundamental form of $M^{n}$. The length of the second fundamental form at $x$ is given by

$$
|\sigma|^{2}(x)=\operatorname{trace} A_{\eta(x)}^{2} .
$$

If $\lambda_{1}(x) \leq \lambda_{2}(x) \leq \cdots \leq \lambda_{n}(x)$ are the principal curvatures of $M^{n}$ at $x \in W$, we have that

$$
|\sigma|^{2}(x)=\sum_{i=1}^{n} \lambda_{i}^{2}(x) .
$$

Given hypersurfaces $M_{1}^{n}$ and $M_{2}^{n}$ of $N^{n+1}$ with $T_{p} M_{1}=T_{p} M_{2}$ (tangent at $p$ ), parametrize $M_{1}^{n}$ and $M_{2}^{n}$ as in (1) obtaining correspondent functions $\mu^{1}$ and $\mu^{2}$. As in (Fontenele and Silva 2001), we say that $M_{1}^{n}$ remains above $M_{2}^{n}$ in a neighborhood of $p$ with respect to $\eta_{o}$ if $\mu^{1}(x) \geq \mu^{2}(x)$ in a neighborhood of zero.

Following the ideas in (Fontenele and Silva 2001), we obtain the following tangency principle:

THEOREM 1.1. Consider hypersurfaces $M_{1}^{n}$ and $M_{2}^{n}$ of $N^{n+1}$ tangent at $p$ and $\eta_{o}$ a unitary vector normal to $M_{1}^{n}$ at $p$. Denote by $\left|\sigma_{1}\right|^{2}(x)$ and $\left|\sigma_{2}\right|^{2}(x)$ the length of the second fundamental form of respectively $M_{1}^{n}$ and $M_{2}^{n}$ at $x \in W$. Assume that $M_{1}^{n}$ remains above $M_{2}^{n}$ in a neighborhood of $p$ with respect to $\eta_{o},\left|\sigma_{1}\right|^{2}(x) \leq\left|\sigma_{2}\right|^{2}(x)$ in a neighborhood of zero and that the principal curvatures of $M_{2}$ at zero are all positive. Under these conditions, $M_{1}^{n}$ and $M_{2}^{n}$ must coincide in a neighborhood of $p$.

For hypersurfaces with boundaries and as a consequence of proof of Theorem 1.1, we obtain the following tangency principle:

THEOREM 1.2. Let $M_{1}^{n}$ and $M_{2}^{n}$ be hypersurfaces of $N^{n+1}$ with boundaries $\partial M_{1}$ and $\partial M_{2}$ respectively. Suppose that $M_{1}^{n}$ and $M_{2}^{n}$ as well as $\partial M_{1}$ and $\partial M_{2}$ are tangent at $p \in \partial M_{1} \cap \partial M_{2}$ and let $\eta_{o}$ be normal to $M_{1}^{n}$ at $p$. Denote by $\left|\sigma_{1}\right|^{2}(x)$ and $\left|\sigma_{2}\right|^{2}(x)$ the length of the second fundamental form of respectively $M_{1}^{n}$ and $M_{2}^{n}$ at $x \in W$. Assume that $M_{1}^{n}$ remains above $M_{2}^{n}$ in a neighborhood of $p$ with respect to $\eta_{o},\left|\sigma_{1}\right|^{2}(x) \leq\left|\sigma_{2}\right|^{2}(x)$ in a neighborhood of zero and that the principal curvatures 
of $M_{2}$ at zero are all positive. Under these conditions, $M_{1}^{n}$ and $M_{2}^{n}$ must coincide in a neighborhood of $p$.

When the ambient is the $(n+1)$-dimensional Euclidean space and the hypersurfaces $M_{1}^{n}$ and $M_{2}^{n}$ have the same constant length of the second fundamental form, Theorems 1.1 and 1.2 are the analogous of the well known maximum principles for hypersurfaces with the same constant $k$-mean curvature in the Euclidean space.

For enunciate our geometric applications let us fix some notation. Given an oriented hypersurface $M^{n}$ of the $(n+1)$-dimensional Euclidean space $\mathbb{R}^{n+1}$, the $k$-mean curvature $H_{k}(p)$ of $M^{n}$ at $p$ is given by

$$
H_{k}(p)=\frac{1}{\left(\begin{array}{l}
n \\
k
\end{array}\right)} \sum_{i_{1}<i_{2}<\cdots<i_{k}} \lambda_{i_{1}} \lambda_{i_{2}} \ldots \lambda_{i_{k}},
$$

where $\lambda_{1} \leq \lambda_{2} \leq \cdots \leq \lambda_{n}$ are the principal curvatures of $M^{n}$ at $p$. In particular, $H_{1}(p)$ is denoted by $h(p)$ and called the mean curvature of $\mathrm{M}$ at $p$ and $H_{2}(p)$ is denoted by $R(p)$ and called the scalar curvature of $\mathrm{M}$ at $p$.

The Ricci curvature of $M^{n}$ at a point $p$ in the direction of an unitary vector $v$ is given by

$$
\operatorname{Ric}_{p}(v)=\frac{1}{n-1} \sum_{i=2}^{n} K\left(v, w_{i}\right),
$$

where $w_{1}=v, w_{2}, \ldots, w_{n}$ is an orthonormal basis of $T_{p} M$ and $K\left(v, w_{i}\right)$ is the sectional curvature of the plane generated by $v$ and $w_{i}$.

Definition 1.3. If $U$ is an open subset of the Euclidean space $\mathbb{R}^{n+1}$, we define

$$
\rho_{U}:=\sup \left\{r>0, \text { such that } \bar{U} \text { contains a closed ball in } \mathbb{R}^{n+1} \text { of radius } r\right\}
$$

where $\bar{U}$ denotes the closure of $U$ in $\mathbb{R}^{n+1}$.

CONDITION I. $M^{n}$ is a complete connected euclidean hypersurface that splits $\mathbb{R}^{n+1}$ into two disjoint regions of which $M^{n}$ is the common boundary.

THEOREm 1.4. Suppose that $M^{n}$ satisfies Condition I. Assume further that $|\sigma|^{2} \geq \frac{n}{\lambda^{2}}$ and $|h|>\frac{n-2}{n \lambda}$ over $M^{n}$, where $\lambda$ is a positive constant. Under these conditions, if we denote by $U$ the component of $\mathbb{R}^{n+1} \backslash M$ that contains the normals for which $h$ is positive, then $\rho_{U} \leq \lambda$. Moreover, if $U \cup M$ contains a closed ball of radius $\lambda$ then $M^{n}$ coincides with a sphere in $\mathbb{R}^{n+1}$ of radius $\lambda$.

Corollary 1.5. Suppose that $M^{n}$ satisfies Condition I. Assume further that $|\sigma|^{2} \geq \frac{n}{\lambda^{2}}$ and $R>\frac{n-4}{n \lambda^{2}}$ over $M^{n}$, where $\lambda$ is a positive constant. Then, the mean curvature function $h$ is positive for some suitable orientation of $M^{n}$ and, denoting by $U$ the component of $\mathbb{R}^{n+1} \backslash M$ that contains the normals for which $h$ is positive, we have $\rho_{U} \leq \lambda$. Moreover, if in the closure of $U$ there exists a closed ball of radius $\lambda$, then $M^{n}$ must be a sphere in $\mathbb{R}^{n+1}$ of radius $\lambda$. 
Corollary 1.6. Suppose that $M^{n}$ satisfies Condition I and that $\left|H_{k}\right| \geq \frac{1}{\lambda^{k}}$ on $M^{n}$ for some $k, 1 \leq k \leq n$, and some positive constant $\lambda>0$. For $k \geq 2$, assume further that there exists at least one point in $M^{n}$ where the second fundamental form is definite. Then there exists a component $U$ of $\mathbb{R}^{n+1} \backslash M$ such that $\rho_{U} \leq \lambda$. Moreover, if $U \cup M$ contains a closed ball of radius $\lambda$, then $M^{n}$ coincides with a sphere in $\mathbb{R}^{n+1}$ of radius $\lambda$.

Theorem 1.7. Suppose that $M^{n}$ satisfies Condition I and is oriented. Assume that $h \geq 0$ and that $|\sigma|^{2} \geq \frac{n}{\lambda^{2}}$ over $M^{n}$, where $\lambda$ is a positive constant. Assume further that $n \geq 3$ and $\operatorname{Ric}_{M} \geq-\frac{1}{(n-1) \lambda^{2}}$. Then, if we denote by $U$ the component of $\mathbb{R}^{n+1} \backslash M$ that contains the normals, we have $\rho_{U} \leq \lambda$. Moreover, if $U \cup M^{n}$ contains a closed ball of radius $\lambda$ then either $n$ must be even or $M^{n}$ coincides with a sphere in $\mathbb{R}^{n+1}$ of radius $\lambda$.

COROLlary 1.8. Suppose that $M^{n}$ satisfies Condition I and that $|\sigma|^{2} \geq \frac{n}{\lambda^{2}}$ over $M^{n}$, for some positive constant $\lambda$. Consider also that $\operatorname{Ric}_{M}>-\frac{1}{(n-1) \lambda^{2}}$. Then, the mean curvature function $h$ is positive for some suitable orientation of $M^{n}$ and denoting by $U$ the component of $\mathbb{R}^{n+1} \backslash M$ that contains the normals for which $h$ is positive, we have $\rho_{U} \leq \lambda$. Furthermore, if in the closure of $U$ there exists a closed ball of radius $\lambda$, then $M^{n}$ must be a sphere in $\mathbb{R}^{n+1}$ of radius $\lambda$.

In the following result, $M^{n}$ is a connected and complete manifold isometrically immersed in $\mathbb{R}^{n+1}$.

Corollary 1.9. Assume that $K_{M} \geq 0$ and $|\sigma|^{2} \geq \frac{n}{\lambda^{2}}$ over $M^{n}$, for some positive constant $\lambda$. If $M^{n}$ is not compact, assume further that there exists at least one point in $M^{n}$ where all sectional curvatures are positive. Then there exists a component $U$ of $\mathbb{R}^{n+1} \backslash M$ such that $\rho_{U} \leq \lambda$. Moreover, if $U \cup M$ contains a closed ball of radius $\lambda$ then $M^{n}$ coincides with a sphere in $\mathbb{R}^{n+1}$ of radius $\lambda$.

\section{SKETCH OF PROOF OF THEOREM 1.1}

Fix an orthonormal basis $e_{1}, e_{2}, \ldots, e_{n}$ in $T_{p} M_{1}=T_{p} M_{2}$ and introduce coordinates, for $x$ in $T_{p} M_{1}$, setting $x=\sum_{i=1}^{n} x_{i} e_{i}$. As in (1), parametrize $M_{1}$ and $M_{2}$ in a neighborhood of $p$ by respectively $\varphi^{1}$ and $\varphi^{2}$, obtaining respectively functions $\mu^{1}$ and $\mu^{2}$. Let $\eta^{l}: W \rightarrow T_{\varphi^{l}(W)}^{\perp} M_{l}, l=1,2$, be a local orientation of $M_{l}$ with $\eta^{l}(0)=\eta_{o}$ and denote by $A_{\eta^{l}(x)}$ the second fundamental form of $M_{l}$ in the direction $\eta^{l}(x)$. Denote by $\varphi_{i}^{l}(x)$ the vector $\frac{\partial \varphi^{l}}{\partial x_{i}}(x)$ and by $A^{l}(x)$ the matrix of $A_{\eta^{l}(x)}$ in the basis $\varphi_{i}^{l}(x), 1 \leq i \leq n$. In (Fontenele and Silva 2001), it is proved the existence of a matrix valued function $\tilde{A}$ defined in $\mathbb{R}^{\frac{n(n+1)}{2}+n} \times \mathcal{N}$, where $\mathcal{N}$ is a connected open set of $\mathbb{R}^{n+1}$ containing the origin, such that

$$
\tilde{A}\left(\mu_{i j}^{l}(x), \mu_{i}^{l}(x), \mu^{l}(x), x\right)=A^{l}(x), x \in W, l=1,2,
$$

where $\left(\mu_{i j}^{l}(x), \mu_{i}^{l}(x), \mu^{l}(x), x\right), 1 \leq i \leq j \leq n$, is a point of $\mathbb{R}^{d}, d=\frac{n(n+1)}{2}+2 n+1$. We write an arbitrary point of $\mathbb{R}^{d}$ as $\left(r_{i j}, r_{i}, z, x\right), 1 \leq i \leq j \leq n$, and $x=\left(x_{1}, \ldots, x_{n}\right)$. Define a function $\Phi: \mathbb{R}^{\frac{n(n+1)}{2}+n} \times \mathcal{N} \rightarrow \mathbb{R}$ by

$$
\Phi=\operatorname{trace} \tilde{A}^{2}
$$


Now using the derivatives of $\tilde{A}$, with respect to the $r_{k l}$ 's, given in (Fontenele and Silva 2001), we obtain that

$$
\sum_{k \leq l=1}^{n} \frac{\partial \Phi}{\partial r_{k l}}\left((1-t) \mu_{i j}^{2}(0)+t \mu_{i j}^{1}(0), 0,0,0\right) \xi_{k} \xi_{l}=2\left\langle A^{t}(0) \xi, \xi\right\rangle
$$

where $A^{t}(0)=\tilde{A}\left((1-t) \mu_{i j}^{2}(0)+t \mu_{i j}^{1}(0), 0,0,0\right)$. Using that $M_{1}^{n}$ remains above $M_{2}^{n}$ in a neighborhood of $p$ with respect to $\eta_{o},\left|\sigma_{1}\right|^{2}(x) \leq\left|\sigma_{2}\right|^{2}(x)$ in a neighborhood of zero and that the principal curvatures of $M_{2}$ at zero are all positive, one can prove that $\Phi$ is elliptic in $\left((1-t) \mu_{i j}^{2}(0)+\right.$ $\left.t \mu_{i j}^{1}(0), 0,0,0\right)$ for $t \in[0,1]$, and, restricting $W$ if necessary, conclude that $\Phi$ is elliptic with respect to the functions $(1-t) \mu^{2}+t \mu^{1}, t \in[0,1]$ (see Fontenele and Silva 2001).

Recalling that $\left|\sigma_{l}\right|^{2}(x)=\operatorname{trace}\left[A^{l}(x)\right]^{2}$, it follows from (2), (3) and our assumptions that

$$
\Phi\left(\mu_{i j}^{2}(x), \mu_{i}^{2}(x), \mu^{2}(x), x\right)=\left|\sigma_{2}\right|^{2}(x) \geq\left|\sigma_{1}\right|^{2}(x)=\Phi\left(\mu_{i j}^{1}(x), \mu_{i}^{1}(x), \mu^{1}(x), x\right), x \in W .
$$

Now the conclusion of the theorem is obtained from the following maximum principle (Alexandrov 1962):

MaXimum Principle. Let $f, g: U \rightarrow \mathbb{R}$ be $C^{2}$-functions defined in an open set $U$ of $\mathbb{R}^{n}$ and let $\Phi: \Gamma \subset \mathbb{R}^{d} \rightarrow \mathbb{R}$ be a function of class $C^{1}$. Suppose that $\Phi$ is elliptic with respect to the functions $(1-t) f+t g, t \in[0,1]$. Assume also that

$$
\Phi\left(f_{i j}(x), f_{i}(x), f(x), x\right) \geq \Phi\left(g_{i j}(x), g_{i}(x), g(x), x\right), \forall x \in U,
$$

and that $f \leq g$ on $U$. Then, $f<g$ on $U$ unless $f$ and $g$ coincide in a neighborhood of any point $x_{o} \in U$ such that $f\left(x_{o}\right)=g\left(x_{o}\right)$.

Now we will prove Theorem 1.7 for give an idea of how one can use Theorem 1.1 to obtain geometric applications.

\section{PROOF OF THEOREM 1.7}

Proof of Theorem 1.7. Consider in $\mathbb{R}^{n+1}$ an arbitrary closed ball $\mathbb{B}_{r}\left[p_{o}\right]$, centered at $p_{o}$ and radius $r$, contained in $U \cup M$. Move $\mathbb{B}_{r}\left[p_{o}\right]$ until its boundary $\mathbb{S}_{r}\left[p_{o}\right]$ touches $M^{n}$ the first time. Let $p$ be a tangency point between $M^{n}$ and $\mathbb{S}_{r}\left[p_{o}\right]$. Denote by $\lambda_{1} \leq \lambda_{2} \leq \cdots \leq \lambda_{n}$ the principal curvatures of $M^{n}$ at $p$. It is well known that $\lambda_{i} \leq \frac{1}{r}$ for all $i$. Since $\lambda_{1}+\lambda_{2}+\cdots+\lambda_{n} \geq 0$ by assumption, we have

$$
\lambda_{1}+\lambda_{2}+\cdots+\widehat{\lambda_{i}}+\cdots+\lambda_{n} \geq-\lambda_{i}
$$

where $\widehat{\lambda_{i}}$ means that $\lambda_{i}$ has been omitted on the sum. Therefore, for a negative $\lambda_{i}$, we deduce that

$$
\frac{1}{\lambda^{2}} \geq-(n-1) \operatorname{Ric}\left(e_{i}\right)=-\lambda_{i}\left(\lambda_{1}+\lambda_{2}+\cdots+\widehat{\lambda_{i}}+\cdots+\lambda_{n}\right) \geq \lambda_{i}^{2}
$$


where $e_{i}$ stands for an unitary eigenvector with eigenvalue $\lambda_{i}$. The above inequality, gives $-\frac{1}{\lambda} \leq$ $\lambda_{i}<0$ for a negative $\lambda_{i}$. We consider two possibilities:

P.1. There exists at $p$ at least one negative $\lambda_{i}$. Denoting by $t$ the number of $\lambda_{i}$ 's that are negative and using our assumption on the length of the second fundamental form, we get

$$
\frac{n}{\lambda^{2}} \leq \sum_{j=1}^{t} \lambda_{j}^{2}+\sum_{j=t+1}^{n} \lambda_{j}^{2} \leq \frac{t}{\lambda^{2}}+\frac{n-t}{r^{2}} .
$$

Thus, $\lambda \geq r$. In case $r=\lambda$, we have that $\lambda_{1}=\lambda_{2}=\cdots=\lambda_{t}=-\frac{1}{\lambda}$ and $\lambda_{t+1}=\cdots=\lambda_{n}=\frac{1}{\lambda}$. Since $h \geq 0$, we obtain that $n \geq 2 t$. On the other hand, we have

$$
\frac{1}{\lambda^{2}} \geq-(n-1) \operatorname{Ric}\left(e_{1}\right)=-\lambda_{1}\left(\lambda_{2}+\cdots+\lambda_{n}\right)=\frac{n-2 t+1}{\lambda^{2}},
$$

which implies that $n \leq 2 t$. Hence, $n=2 t$ and $n$ is even. Notice also that $M^{n}$ is minimal at $p$ and the Ricci curvature at $p$ is constant and equal to $-\frac{1}{(n-1) \lambda^{2}}$.

P.2. At $p$, all $\lambda_{i}{ }^{\prime} s$ are nonnegative. In this case,

$$
\frac{n}{\lambda^{2}} \leq \lambda_{1}^{2}+\lambda_{2}^{2}+\cdots+\lambda_{n}^{2} \leq \frac{n}{r^{2}}
$$

Thus $\lambda \geq r$. If $r=\lambda$, it follows easily that $\lambda_{1}=\lambda_{2}=\cdots=\lambda_{n}=\frac{1}{\lambda}$. Using Theorem 1.1 and noting that $\frac{n}{\lambda^{2}}$ is the constant value of the length of the second fundamental form of a sphere having radius $r$, we obtain that $M^{n}$ and $\mathbb{S}_{r}\left[p_{o}\right]$ coincide in a neighborhood of $p$. By an argument of connectness, we conclude that $M^{n}$ is equal to $\mathbb{S}_{r}\left[p_{o}\right]$.

REMARK 3.1. We point out that Corollary 1.6 extends to complete hypersurfaces Theorem 1.3 in (Fontenele and Silva 2001) and that its hypothesis are stronger than those in Theorem 1.4. In fact, for $k=1$, this follows from the well known inequality $|\sigma|^{2} \geq n h^{2}$ and, for $k>1$, this follows from Lemma 1 in (Montiel and Ros 1991). Furthermore, the estimate for $\rho_{U}$ in Theorem 1.4 improves the one given by Corollary 1.6. For if inf $|h|=\frac{1}{H_{o}}>0$ and inf $|\sigma|^{2}=\frac{n}{\lambda_{o}^{2}}$ with $|h|>\frac{n-2}{n \lambda_{o}}$, the upper bound given for $\rho_{U}$ in Theorem 1.4 is $\lambda_{o}$ and the one given by Corollary 1.6 is $H_{o}$. That $\lambda_{o} \leq H_{o}$ follows from the well known inequality $|\sigma|^{2} \geq n h^{2}$. For example, in the cilinder $C=\mathbb{S}^{1} \times \mathbb{R}$ in $\mathbb{R}^{3}$, oriented by the normals pointing to the component $U$ of $\mathbb{R}^{3} \backslash C$ containing the origin, where $\mathbb{S}^{1}$ is the unitary circle, the mean curvature and length of the second fundamental form are given respectively by $\frac{1}{2}$ and 1 . The estimate given by Theorem 1.4 is $\rho_{U} \leq \sqrt{2}$, while the estimate given by Corollary 1.6 is $\rho_{U} \leq 2$.

\section{RESUMO}

Neste trabalho nós provamos um princípio de tangência (veja Fontenele and Silva 2001) para hipersuperfícies de um espaço ambiente arbitrário e relacionado com o comprimento da segunda forma fundamental. Como aplicações geométricas, nós fazemos estimativas dos raios das bolas contidas em uma determinada 
componente conexa do complemento de uma hipersuperfície completa do espaço Euclidiano, generalizando e melhorando estimativas de raios análogas obtidas por Blaschke, Koutroufiotis e os autores. O fato básico estabelecido aqui é que um determinado operador é elíptico nos pontos onde a segunda forma fundamental é positiva definida.

Palavras-chave: hipersuperfícies, princípio de tangência, segunda forma fundamental, bolas, estimativas de raios.

\section{REFERENCES}

Alexandrov AD. 1962. Uniqueness theorems for surfaces in the large I-V, Amer Math Soc Transl, Ser. 2, 21: 341-416.

BlaschKe W. 1956. Kreis und Kugel. 2. Auflage, Berlin.

Fontenele F And Silva SL. 2001. A tangency principle and applications, Illinois J Math, 45: 213-228.

Koutroufiotis D. 1973. Elementary geometric applications of a maximum principle for nonlinear elliptic operators, Arch Math, 24: 97-99.

Montiel S AND Ros A. 1991. Compact hypersurfaces: the Alexandrov theorem for higher order mean curvatures, Differential Geometry (Lawson B and Tenenblat K, eds.), 52, Pitman Monographs, Essex, Longman: 1-13. 